\title{
Purposeful Incorporation of Patient Narratives in the Medical Record in the Netherlands
}

\author{
Hilde Luijks, MD, PhD, Kees van Boven, MD, PhD, Tim olde Hartman, MD, PhD, \\ Annemarie Uijen, $M D$, PhD, Chris van Weel, MD, PhD, and Henk Schers, MD, PhD
}

Introduction: Structuring patient and practice data into episodes formed the foundation of the earliest evidence base of family medicine. We aim to make patients' narratives part of the evidence base for family medicine by incorporating coded and structured information on the patient's reason to visit the family physician (FP) and adding the patient's personal and contextual characteristics to routine registration data. This documentation allows studies of relations between morbidity and elements of the patient story, providing more insight into the range of problems presented to primary care and in the patient-centeredness applied by FPs.

Methods: The Dutch Practice-Based Research Network (PBRN), named FaMe-Net, is the world's oldest PBRN. Seven Dutch family practices provide regular primary care and participate in the PBRN. It contains all morbidity data of the approximately 40,000 listed patients $(308,000$ patient-years and 2.2 million encounters from 2005 until 2019). All information belonging to 1 health problem is ordered in 1 episode. Morbidity (diagnoses), reasons for encounter (RFE), and interventions are coded according to the International Classification of Primary Care (ICPC-2). Registration occurs within the electronic health record (EHR), specially designed to facilitate the extensive registration for the PBRN. Since 2016, the network expanded routine registration with the duration of symptoms and coded personal and contextual characteristics (eg, country of birth, level of education, family history, traumatic events) obtained through the self-reported 'context survey' of listed patients. These data are added to the EHR. Registered data are extracted from the EHR and processed for scientific research.

We present data on the differences in RFEs of the most prominent symptoms of COVID-19 between 2019 and 2020; the relation between the diagnosis of pneumonia and presentation of the symptom 'cough,' and how personal determinants influence the chances of final diagnoses. Lastly, we show the relation of selfreported abuse with patient's contact frequency and psychosocial problems.

Results: Prompt introduction of registration rules brought insight into COVID-19-related symptoms early in the pandemic. In March 2020, symptoms related to COVID-19 were presented more often than in March 2019. Chronic conditions and prevention showed a collapsing contact frequency. Telephone, email, and video consultations increased from $31 \%$ to $53 \%$.

Episodes of pneumonia most frequently started with the RFE 'cough.' A combination of 'cough' and 'fever' as RFE increases the likelihood of pneumonia, as does cough in the presence of comorbid COPD among older men. The prevalence of pneumonia is higher among patients with low socioeconomic status.

Discussion: The Dutch PBRN FaMe-Net has started to add elements of patients' narratives and context to decades of morbidity registration, creating options for a scientific approach to primary care's core values. Assumptions of 'pre/post chances' of the final diagnosis, already existing implicitly in FPs minds, can be elaborated and quantified by investigating the associations between multiple registered variables, including parts of patients' 'stories.' This way, we aim to make visible what is intuitively already known by FPs. (J Am Board Fam Med 2021;34:709-723.)

Keywords: Comorbidity, Epidemiology, Family Medicine, Longitudinal Research, Narrative Medicine, Primary Health Care, Social Determinants of Health

\section{Introduction}

Family medicine adds value to the health care system as a whole. Its core values are based on a

This article was externally peer reviewed.

Submitted 23 November 2020; revised 8 April 2021; accepted 14 April 2021.

From the Department of Primary and Community Care (117-ELG), Radboud Institute for Health Sciences, profound knowledge of epidemiology and continuous and personal relationships with patients. ${ }^{1,2}$ This article aims to show the value of structuring

Radboud University Medical Center, Nijmegen, The Netherlands.

Funding: None.

Conflict of interest: None.

Corresponding author: Hilde Luijks, MD, $\mathrm{PhD}$, Radboudumc, Nijmegen, $6500 \mathrm{HB}$, Netherlands, Phone: +31 633057115 (E-mail: Hilde.Luijks@radboudumc.nl). 
patient and practice data into episodes. Episodebased epidemiology was the foundation for the earliest evidence base of family medicine, ${ }^{3,4}$ and may be considered a foundational method for family physicians worldwide. Its richness can be expanded by incorporating biographical, contextual, and clinical data, ${ }^{4-7}$ thus making patients' narratives part of the evidence base for family practice.

The Dutch Practice-Based Research Network (PBRN) 'Family Medicine Network (FaMe-Net),' the world's oldest PBRN, has a long history of systematically recording all morbidity presented to a family physician (FP), categorized in episodes. ${ }^{4-7}$ FaMe-Net is the continuation of decades of primary care research. In this article, we will describe the network's methods of data collection and introduce its efforts to capture core values of family medicine in its expansion of the collection of data: patients' characteristics, contextual information, and the reason for encounter, which is the patient's verbalized reason why to contact the FP in the opening of every consultation. The novelty and relevance of adding these data to the episode registration lie in the complementarity of data, allowing studies of mutual relations between registered variables, such as morbidity (diagnoses), duration of symptoms, and elements of 'the patient's story' (RFE and context). They bring more insight into the full range of presented morbidity to primary care, with relevance for clinical training.

Questions about the evidence for core values in family practice are still unanswered to a large extent. What can be learned from the distributions of the reason for encounter? How can FPs use contextual knowledge in daily practice for the patients' benefit? In addition, questions about how reasons for encounter and final diagnoses relate to patients' characteristics and context are largely unknown.

This article aims to show examples of how routine registration of new data in a research and registration network enables research into the relation between biopsychosocial determinants and morbidity, policy, and outcomes. We will show how the Coronavirus disease 2019 (COVID-19) pandemic led to changes in respiratory symptoms presented to the FP, how pneumonia is intertwined with RFE, and other determinants (sex, age, educational level, comorbidity). Lastly, we will demonstrate how contextual data relate to the prevalence of health problems and contact frequency.

\section{Methods}

\section{Setting}

Appendix 1 provides background information on the network, explaining FaMe-Net's evolution from its predecessor networks. Moreover, it shows its embedding in the Dutch health care context in which family medicine has a pivotal position. Almost all Dutch inhabitants are listed with 1 family practice, which serves as the first point of access to health care. It provides care for the large majority of health problems and coordinates access to specialized care. FaMe-Net FPs provide regular primary care to their listed patients. Registering for the PBRN occurs simultaneously. FaMe-Net does not apply a focus on specific diseases or patient groups. ${ }^{8}$ Data are collected longitudinally.

\section{Structured Data Registration Episode of Care (EoC)}

In episode of care registration, all information belonging to 1 health problem is ordered in 1 episode and can be traced back. ${ }^{9,10}$ An EoC is defined as 'a health problem in an individual from the first until the last encounter with a health care provider'. Dutch FPs have an overview of all medical data of listed patients since all primary care is provided by the patient's own FP (practice). All information, including hospital/specialist reports, is sent to the FP. FPs link this information to an EoC. This documentation is facilitated using an electronic health record (EHR) for all listed patients. FPs from $\mathrm{FaMe}-\mathrm{Net}$ are trained to order all data carefully into an Episodes of Care (EoC) structure. An example of the evolution from the symptom fatigue to the end diagnosis of colorectal carcinoma is shown in Box 1 and Figure 1.

\section{ICPC}

All presented symptoms, complaints, diseases, and problems are classified by the $\mathrm{FP}$ in accordance with the International Classification of Primary Care (ICPC-2) at the highest level of accuracy and understanding. All interventions and processes are also coded with ICPC-2. These include referral to primary or secondary care professionals, diagnostic 
Box 1: Episodes of Care (EoC) registration: An example of the evolution of a diagnosis

The EoC title (the diagnosis) can be modified along the track. For example, when a patient enters the consultation room uttering the symptom fatigue, this symptom will be coded as RFE. The diagnosis will be fatigue as well, and the $\mathrm{EoC}$ title will be fatigue at the start of this episode. Later, because of a low hemoglobin found with laboratory testing, the EoC title fatigue will be changed into iron deficiency anemia. However, when it appears that this iron deficiency anemia is caused by a colon carcinoma (found after colonoscopy) then the EoC title (the diagnosis) will be changed into colon carcinoma. As a result of such registration in an EoC structure we are able to review how EoC started (in this example with the RFE fatigue) and how it evolved (in this example via initial diagnoses fatigue and iron deficiency anemia to colon carcinoma).

imaging, laboratory testing, and therapeutic interventions such as medication, vaccination, or surgical procedure. Prescriptions are coded according to the Anatomic Therapeutic Chemical (ATC) coding system maintained by the World Health Organization. Transfer to ICPC version 3 is planned soon after its release in December 2020, allowing for the additional recording of functioning (activities and participation) and personal preferences linked to morbidity.

\section{Reason for Encounter (RFE)}

An important registration issue is the Reason For Encounter. Patients normally start the consultation with a spontaneous statement on why they visit the doctor. This literal articulation of why to consult, expressed by the patient, is taken verbatim by the doctor. It reflects the initial presentation of an illness at the physician's office. It precedes the

Figure 1. Episodes of Care registration as an example of the evolution of a diagnosis.

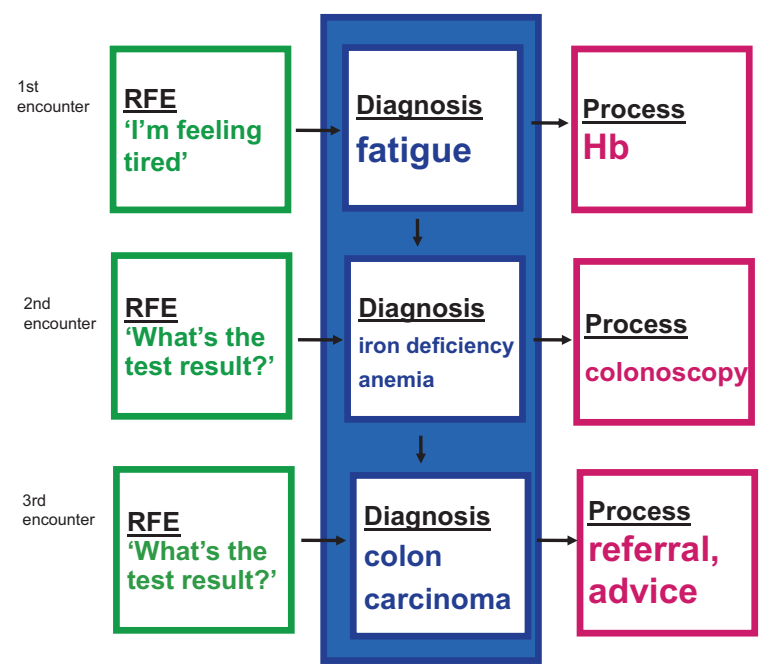

interaction between patient and FP. The RFE(s) can be presented as a symptom (eg, abdominal pain, rash) but also as a self-diagnosed disease ('I've got the flu') or a request for a particular intervention. FaMe-Net FPs carefully register RFEs regardless of diagnosis. The coding of RFE enables research that studies associations between RFE and final diagnosis. RFEs in themselves have proven to have important prognostic value, for example, in diagnosing cancer. ${ }^{11-13}$ Since 2016, the network has started to code the duration of symptoms as well. FPs will classify the duration of a symptom in hours, days, weeks, months, or years at the first encounter of an episode for every new RFE. Patients often do not exactly state the duration of their symptoms in the opening sentence. FPs record RFE duration based on information provided by the patient during the encounter.

\section{Health care Trajectories}

FaMe-Net's registration allows studying health care trajectories, including all diagnostic and therapeutic interventions, the number of encounters, or the elapsed time between initial presentation and final diagnosis. In this way, it can be studied what happened within specific EoCs, or within a period (eg, 1 calendar year). The larger part of the registered encounters concerns consultations during office hours. Diagnoses reported during home visits, telephone or e-mail consultations (by the FP or the practice assistant), out-of-hours consultations, and administrative contacts (specialist letters) also contribute to the registered morbidity.

\section{Contextual and Personal Characteristics}

In addition, since 2016, FaMe-Net has started to collect contextual and personal characteristics of all the listed adult patients in a structured way. For this 
purpose, these patients are invited by their $\mathrm{FP}$ to complete a so-called online 'context survey' including personal (demographic) characteristics such as country of birth of the patient and the patients' parents, level of education, working status and working hours, and contextual characteristics such as traumatic life events including abuse (sexual, physical or psychological violence) and intoxications, as well as information on the family history for diabetes, cardiovascular disease and several cancer types. ${ }^{14}$ (Appendix 2) Completed surveys are automatically added to patients' EHR and visible for their FP in daily practice. FaMe-Net asks patients to check and update the context surveys on a 2-yearly basis. Non-responders are sent a reminder to complete the survey. The socioeconomic status (SES) is derived from the highest completed educational level, in accordance with Statistics Netherlands' advice. ${ }^{15}$ The percentage of adult patients that have completed the context survey has increased to $55 \%$ at the end of 2020 .

\section{Data Management and Quality}

Registration of all items occurs within the EHR specially designed to facilitate this extensive registration for the PBRN. It fully functions as EHR and includes functionalities to refer patients to secondary or other primary care settings, prescribe medication, and finance the health care process. The PBRN is a collaboration between Radboudumc and the affiliated practices. All data registered for $\mathrm{FaMe}-\mathrm{Net}$ are extracted from the EHR and stored de-identified in the Radboudumc Technology Center Health Data of the Radboud University Medical Center. RTC processes and manages the data for scientific research and secures the data and the privacy of the involved patients. Patients listed in the practices may opt-out for the extraction of their data for research. This procedure complies with all Dutch privacy legislation. Data are not all open-source data: only affiliated researchers with permission to study these data have access. FaMe-Net provides access to an open-source website with some of the extracted data (www. famenet.nl).

FaMe-Net performs systematic quality checks of the stored data and provides registration feedback to FPs. Uniformity of coding is achieved through continuous online training (coding uniformity) and quality control programs for FPs (in training), practice assistants, and practice nurses.

\section{Analysis and Presentation of Data}

The complete dataset until the end of 2018 includes over 278,000 patient-years with more than 2.2 million encounters. Prevalence and incidence are expressed per 1,000 patient-years with methods identical to those used by Netherlands Institute for Health Services Research. ${ }^{16}$

For this article, we show data of the COVID-19 pandemic. After the first case in the Netherlands, FaMe-Net started registering specific codes for COVID-19 (ICPC R83), following the ICPC-manual. We analyzed the incidence of RFEs of the most prominent symptoms of COVID-19 (ie, cough, shortness of breath, tiredness, sore throat, common cold, and fever), comparing the period of February-May 2019 with February-May 2020. Furthermore, we analyzed how this sudden outbreak impacted the number and types of encounters by comparing 2019 and 2020 data on FaMe-Net's most common health problems: hypertension, diabetes, mental health problems, otitis, fatigue, and preventive counseling. ${ }^{17}$

Next, we will present epidemiologic data on presentation of 'cough' (RFE: ICPC R05), which was the most commonly presented symptom in FaMe-Net overall, and its relation to the EoC 'pneumonia' (ICPC R81). We analyzed associations with education levels and used the Bayesian network approach to illustrate how (combinations of) previously made diagnoses determine probability distributions of new diagnoses on the presentation of 'cough.' It uses variables with probabilities for each of their values. Figure 2 visualizes how registered elements can influence end diagnosis probabilities.

Figure 2. Influence of registered variables on the probability of the end diagnosis. The total number of patient-years was 278,126 in the 2005-2019 dataset. Socioeconomic status was known for $36 \%(n=1576)$ of patients with an Episode of Care of pneumonia. Abbreviation: RFE, Reason for Encounter.

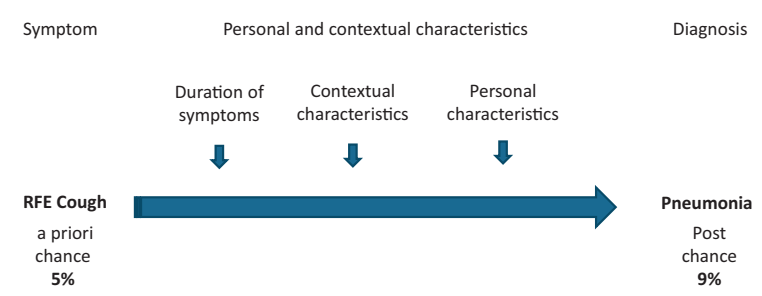


Finally, we describe 2 different examples illustrating relations between context survey information and outcomes. For 'reported abuse,' we explored associations with patient's contact frequency and psychological and social problems. For 'a family history on cardiovascular disease,' we studied the presence of 3 essential items in the EHR needed to calculate the cardiovascular disease (CVD) risk: smoking status, systolic blood pressure (SBP), and total cholesterol/high-density lipoprotein (HDL) cholesterol ratio.

\section{Results}

\section{Significance of PBRNs in Understanding New Health Problems: The COVID-19 Example}

In an earlier article, we described how our network was able to react very quickly to the upcoming COVID-19 crisis by introducing definitions and coding rules. We were able to code COVID-19 from day 1 based on presenting clinical symptoms before polymerase chain reaction (PCR) testing became available to Dutch FPs. In March 2020, symptoms related to COVID-19 were presented far more often than in March 2019, and COVID-19 became the commonest respiratory tract-related RFE. FaMe-Net's registration showed 558 (clinical) COVID-19 diagnoses on May 31, with only $10 \%$ confirmed by a positive PCR test. The contact frequency with FP remained stable, with a sharp increase in telephone, email, and video consultations from $31 \%$ in 2019 to $53 \%$ in March 2020 $(P<.001)$. The contact frequency for hypertension, diabetes, fatigue, otitis, and prevention plummeted substantially from early March 2020. Mental health problems were presented in comparable numbers. ${ }^{17}$

Figure 3. Age distribution of episodes starting with a reason for encounter of 'cough'. The total number of patient-years was 278,126 in the 2005-2019 dataset.

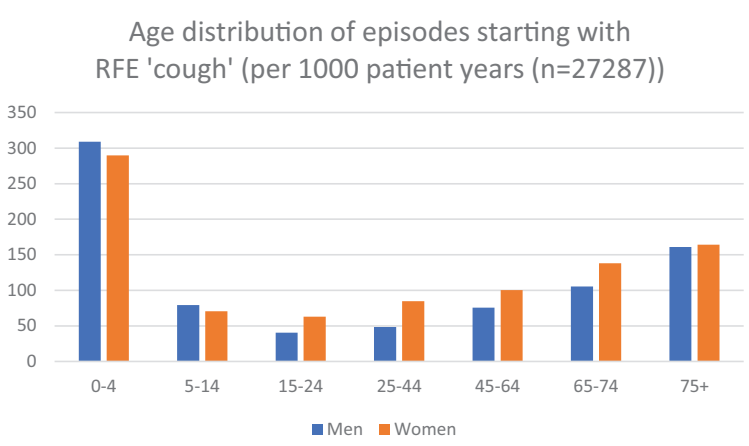

Dataset: 2005-2019. Total number of patient years included: 278,126.

\section{Relations between RFE, Diagnosis, and Other Determinants: Cough and Pneumonia}

The incidence of the symptom 'cough' was 98 per 1000 patient-years. Among the youngest and oldest patients, Episodes of Care started more often with the symptom cough (Figure 3). 'Cough' was seen more frequently in women. Patients with a low educational level presented 'cough' more often than patients with a high educational level (risk ratio [RR], 1.7; 95\% confidence interval [CI], 1.56-1.95). The duration of cough before the first practice visit was most often 2 to $7(32 \%)$ or 7 to 14 days (22\%), with a minority (5\%) reporting cough for 2 days or less.

\section{Final Diagnoses Starting with Presentation of 'Cough'}

New episodes starting with the symptom cough were most often diagnosed as 'cough' (symptom diagnosis [31\%]) and 'acute upper respiratory tract infection' (29\%, Table 1). 'Pneumonia' was most seen in the youngest and oldest age groups presenting with 'cough': 14 and 18 per 1000 patient-years among the 0 to 4 years and 75 year and older age groups, respectively. The end diagnosis 'pneumonia' for patients presenting with 'cough' was made in $6 \%$ overall (Table 1), with slightly higher proportions of pneumonia among children (5 to 14 years: $7 \%$ ) and elderly (65 to 74 years: $8 \%$ ). The prevalence of pneumonia was highest among the eldest (75 years and older) presenting with 'cough': $11 \%$.

Table 1. Final Diagnosis of New Episodes Presented with the Reasons for Encounter of 'Cough'

\begin{tabular}{clc}
\hline ICPC Code & \multicolumn{1}{c}{$\begin{array}{c}\text { Episode Label } \\
\text { (Final Diagnosis) }\end{array}$} & $\begin{array}{c}\text { \% Final } \\
\text { Diagnosis }\end{array}$ \\
\hline R05 & Cough $^{\ddagger}$ & 30.7 \\
R74 & Acute upper respiratory infection $^{\dagger}$ & 28.5 \\
R78 & Acute bronchitis/bronchiolitis & 14.2 \\
R77 & Acute laryngitis/tracheitis & 6.8 \\
R81 & Pneumonia & 6.4 \\
R80 & Influenza & 2.6 \\
R75 & Acute/chronic sinusitis & 2.3 \\
H71 & Acute otitis media/myringitis & 1.1 \\
R96 & Asthma & 1.0 \\
A77 & Viral disease, other/NOS & 0.5 \\
\hline
\end{tabular}

ICPC, International Classification of Primary Care.

* Cough is coded as R05 in the 2005-2019 FaMe-Net dataset.

${ }^{\dagger}$ Total number of patient-years: $278,126$.

${ }^{\ddagger}$ Number of episodes starting with 'cough': 27,287. 
Overall, $38 \%$ of the new episodes starting with a cough had an additional RFE combined with cough. The most common additional RFE was fever (A03: 11\%), followed by dyspnea (R02: 6\%) and throat symptoms (R21: 4\%). The first 2 combinations (cough and fever, cough and dyspnea) increase the proportion of pneumonia as end diagnosis to $19 \%$ and $12 \%$ respectively (compared with $6 \%$ overall with 'cough'), whereas the third combination of RFEs (cough and throat symptoms) drops the chance of pneumonia to $1 \%$.

\section{Episodes of Pneumonia: RFE, Duration, and Health care Trajectory}

Pneumonia had a prevalence of 17.2 per 1000 patient-years. Pneumonia was most seen among the youngest (0 to 4 years) and oldest (65 to 74 years and 75 years and older) age groups; it did not differ significantly between men and women in the total population. Figure 4 shows the variation between age groups, with a significant sex difference, noted only among the eldest patients. For those with a known SES, the prevalence of pneumonia was notably higher among patients with a low SES than a high SES (24 vs 15 per 1000 patient-years, respectively).

EoC with the final diagnosis pneumonia most frequently started with 'cough,' followed by fever, dyspnea and 'Doctor, could I have a pneumonia?' as shown in Table 2. 'Cough' was the commonest $\mathrm{RFE}$ in all age categories, except for the youngest patients (0 to 4 years), in whom the commonest RFE was 'fever.' In $38 \%$ of all episodes of pneumonia starting with RFE 'cough,' this symptom existed between 2 and 14 days. Two days or shorter was

Figure 4. Prevalence of Episodes of Care 'pneumonia' among different age categories. Abbreviation: SES, socioeconomic status.

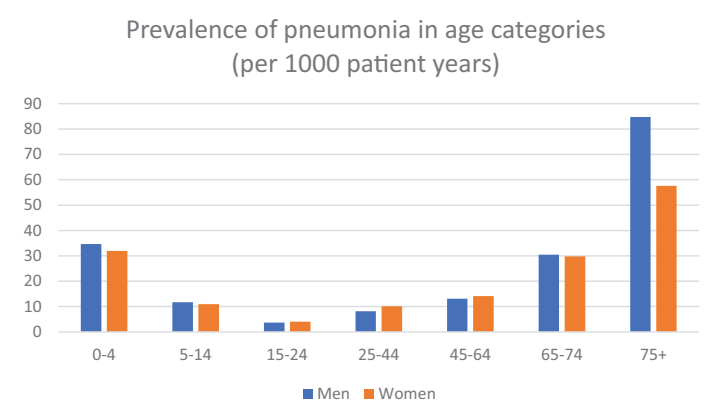

Dataset: 2005-2019. Total number of patient years included: 278,126

SES was known for $36 \%(n=1,576)$ of patients with an Episode of Care of pneumonia reported in $4 \%, 7$ to 14 days in $26 \%$, and longer than 14 days in $32 \%$. An episode of pneumonia often $(66 \%)$ counted 1 or 2 encounters, up till 3 encounters in $80 \%$, and more than 7 encounters in $4 \%$. Pneumonia is a disease that is relatively often first presented 'out-of-hours' (13\%). Most referrals for pneumonia were to pulmonology $(6 \%)$ and internal medicine and pediatrics (both $2 \%$ ).

\section{Influence of Personal Characteristics on the Chance of Having a Pneumonia}

The use of the Bayesian network approach showed that the likelihood of pneumonia among male patients aged 75 years and older consulting with RFE cough was twice as high for persons with previously diagnosed COPD, as compared with $75+$ aged men without COPD. However, for those who additionally visited the FP in the year before with psychological problems (apart from the COPD), the likelihood of pneumonia dropped to $65 \%$ compared with their sex-age matched peers. This outcome resulted in probability distributions of final diagnoses for the specific symptom cough in which adding data (previously made diagnoses) changed the likelihood of final diagnoses considerably.

Table 2. The Commonest Presented Symptoms (Top 10 of Reasons for Encounter) at the Start of an Episode of Pneumonia*

\begin{tabular}{llc}
\hline $\begin{array}{l}\text { ICPC } \\
\text { Code }\end{array}$ & $\begin{array}{c}\text { RFE at the Start of } \\
\text { Pneumonia Episode }^{\dagger}\end{array}$ & $\begin{array}{c}\text { \% Pneumonia Episodes } \\
\text { With This Start RFE }^{\ddagger}\end{array}$ \\
\hline R05 & Cough & 43 \\
A03 & Fever & 27 \\
R02 & Dyspnea & 16 \\
R81 & Pneumonia & 7 \\
A04 & Weakness/tiredness & 3 \\
A05 & Feeling ill & 3 \\
R74 & Acute upper respiratory & 2 \\
& infection & 2 \\
R01 & Pain respiratory system & 1 \\
L04 & Chest symptom/ & \\
& complaint & 1 \\
R80 & Influenza & 14 \\
& Other symptoms & \\
\hline
\end{tabular}

ICPC, International Classification of Primary Care; RFE; reasons for encounter.

*Pneumonia is coded as R81 in the 2005-2019 FaMe-Net dataset.

${ }^{\dagger}$ Number of new episodes of pneumonia: 3988.

${ }^{\ddagger}$ Percentages cumulate to more than 100 since multiple RFEs can be recorded. 
Contextual Information and Health Care Outcomes: Abuse and Family History

Associations between History of Abuse, HelpSeeking Behavior, and Psychological and Social Problems

Approximately $7 \%$ of men and $15 \%$ of women selfreported abuse. It appeared that both men and women who indicated that they did have a history of abuse (1,271 of 11,142 completed questions) contacted the FP 1.5 times as often, compared with patients reporting they $\operatorname{did} \operatorname{not}(P<.001)$. RFEs for patients with a history of abuse were almost twice as often for psychological $(\mathrm{RR}=1.97 ; P<.001)$ and social problems $(\mathrm{RR}=1.93 ; P<.001)$. EoCs of psychological and social problems were also registered almost twice as much for patients with a history of abuse. We observed no differences between men and women.

Family History of CVD and Complete EHR Registration of CVD Risk Factors

A positive family history of CVD was reported by $23 \%$ of patients aged less than 65 years $(n=2142$ of 9372 completed questions). This was not registered in the EHR in $90 \%$ of the cases. We found an odds ratio of 2.83 (95\% CI, 2.53-3.15) for complete data in the EHR on the 3 items smoking status, SBP, and total cholesterol/HDL cholesterol ratio, needed for CVD risk calculation: all items were registered in $35 \%$ of the patients with a positive, versus $16 \%$ with a negative family history. The variables age, presence of other cardiovascular risk factors, presence of CVD, type 2 diabetes, and chronic kidney disease appeared as independent variables increasing chances of complete CVD risk profile. ${ }^{18}$

\section{Discussion}

This article has shown the value of expanded data registration in our professional practice-based research network. This is a first step in making visible what is already intuitively known by many family physicians. The Episode of Care structure, enriched by the structured collection of coded elements of contextual and personal characteristics, is valuable and will give practice-based datasets more predictive power. In essence, it adds parts of patients' narratives to traditional epidemiologic morbidity registration and attempts to build evidence for the core values of family practice. It may also fuel the development of diagnostic support systems in general practice.

The expanded registration increases insight into the coherence between patient's disease, presentation (RFE, duration), personal circumstances, the health care trajectory, and primary care outcomes. Coded data of patient's phrasing of why they consult the FP appeared to have major significance for the final diagnosis. RFEs represent patients' needs when seeking medical help. The importance of applying a patient-centered generalist approach, responding to patients' needs, is intuitively known and acted on by FPs, but the scientific evidence for the relevance of applying this approach is fragmented. ${ }^{19,20}$ When evaluating processes and outcomes of care, more focus could be put on their patient-centeredness and include the RFE. ${ }^{21}$ FaMe-Net's expanded data registration may give explanations why different interventions are conducted for similar diagnoses. Variability may be caused by responsiveness to patients' contextual circumstances or reasons why they consulted the doctor. It builds ways to demonstrate that FPs act patient centered. Knowing the patient and his context, including his health history, personal circumstances, and his narrative story, are all thought to influence help-seeking behavior and predict morbidity patterns. ${ }^{2,22,23}$ Moreover, these factors influence FPs' diagnoses and policy. The impact of narratives on medical practice and patients' lives should be deepened, as also advocated by Narrative Medicine's movement. ${ }^{24}$ FaMe-Net's methodology of recording RFEs by FPs may not be exactly similar to the approach described by the Narrative Medicine movement, ${ }^{23,25}$ but relating RFEs and the self-reported contextual patient characteristics to the morbidity registration gives a tremendous scientific boost to the noted scarcity of studies on the effects of Narrative Medicine on patient's illness experience. ${ }^{24}$ The expanded registration aims to substantiate the many things happening in the consultation room and quantify the relevance of FPs' contextual knowledge of the patient's personal situation that is often not reported systematically.

Our experience and agility as PBRN and the quickly made registration agreements fulfilled the need for primary care data shortly after the COVID-19 outbreak in the Netherlands. The registration, including RFE, provided unique insight into COVID-19 diagnoses and their symptoms in primary care from the beginning of the pandemic, their effects on 
primary care 'consumption,' and the impact of governmental policy.

The predictive value of the RFE for end diagnosis emphasizes the importance of the story the patient tells. It is embedded in the diagnostic challenges that FPs face every day. An RFE of 'fever' or 'cough' is not equal to having fever measured in the office or confirmation of cough as a symptom during history taking. When coded as RFE, these complaints were probably valued by patients as important issues since they utter them as a reason to consult the FP when starting the consultation. It should not be underestimated how patients' stories result in different interventions. A worker fearing lung cancer could have a chest radiograph, and 'reassurance' as intervention, a professor preparing an oral presentation could have codeine prescribed.

We showed the importance of patients' 'stories' in example studies with data on patient's contextual characteristics. A high contact frequency for psychosocial problems should alert FPs to inquire about abuse in both sexes. CVD risk is often unknown and could be made more explicitly visible by the patient's input, leading to completion of relevant measurements in the EHR.

FaMe-Net reports a similar incidence and prevalence of pneumonia as Netherlands institute for health services research. ${ }^{16,26}$ The prevalence of presented symptoms is related to patient characteristics such as age and SES. These characteristics also influence the probabilities of diagnoses, as shown in our examples of pneumonia and cough. Probably, pre/post chances on specific outcomes are roughly known by experienced FPs who know their patients well, integrating all their knowledge of this patient in an unexplicit risk assessment once new consultations with new information start. We have started to make these predictions visible and quantifiable with the Bayesian network approach. This approach elaborates the probability calculations, considering additional personal characteristics. Such analyses may contribute to clinical reasoning in medical training: it is relevant to know that presentation with fever or dyspnea and cough, respectively, triple and double the chances of pneumonia as the final diagnosis. Moreover, more profound predictive knowledge may lead to diagnostic support tools for FPs.

Innovations can augment the possibilities for analysis of individual patient factors in relation to morbidity data. Currently, all data are available but not yet swiftly accessible, requiring ' 1 -by-1' data extractions. FaMe-Net is working on automatizing these operations. Through an updated FaMe-Net website, 'complete' primary care epidemiologic data will be provided, increasing accessibility and availability. Deeper analyses can help find associations currently unknown, predict which patients are at risk of developing specific diseases, and predict disease outcomes in specific patient groups. Bayesian networks have the advantage that they can be explained logically, increasing trustworthiness of the model. ${ }^{27-29} \mathrm{FaMe}-\mathrm{Net}$ aims to develop additional methods using Artificial Intelligence, of great help when investigating multiple relations: between morbidity (final diagnoses), comorbidity, mortality, illness presentation (RFE and duration), and contextual/personal characteristics including intoxications and ethnicity. Our expanded means to describe how morbidity is presented in family medicine form the basis of scientifically grounded family medicine. AI may help quantify assumptions of 'pre/post chances,' already existing implicitly in FPs minds. With gradual growth of the database, knowledge increases. FaMe-Net can bring important contributions to public health by targeting patient education, accounting for individual risk profiles. $^{30}$

\section{Limitations}

The data collected through the FaMe-Net context survey are self-reported. In theory, this information may be incorrectly or inaccurately, although normally, this kind of self-reporting has proven to be valid. Completing the context survey can be demanding for patients, especially for sensitive issues or when explanation is desired. This issue is inherent to data collection through surveys. Patients are notified that they may discuss issues with their FP. Not all patients have completed the context survey data, and contextual data, therefore, do not cover the complete FaMe-Net population. However, we have observed a gradual increase in the response rate among listed patients and consider the response rate high $(>50 \%)$. Lower educated patients and those visiting their FP relatively infrequently are underreported in the current sample, influencing the results. The context survey certainly does not an equal careful history taking on sensitive issues such as abuse. Nevertheless, it brings elements of a patient's narrative story that 
often seem unknown to our data set and makes this knowledge available for research. Patients do not review the RFE as recorded by the FP. RFEs capture an important part of the patient's story-their literal expressed reason to consult-but not necessarily a patient's deepest concern.

\section{Conclusion}

The Dutch PBRN FaMe-Net has expanded its registration routines. The continuous collection of structured data now includes multiple elements on contextual, personal, and narrative aspects, creating options for studying primary care's core values, especially from family practice. In this way, morbidity data can be linked to biopsychosocial determinants and elements of 'patient stories'-making visible and measurable what is intuitively already known by FPs.

FaMe-Net website visitors can get access on request to epidemiologic data on other EoCs, RFEs and interventions (www.famenet.nl).

The authors would like to acknowledge all registering FaMeNet FPs, practice assistants, and practice nurses.

To see this article online, please go to: http://jabfm.org/content/ 34/4/709.full.

\section{References}

1. Phillips RL, Jr., Pugno PA, Saultz JW, et al. Health is primary: family medicine for America's health. Ann Fam Med 2014;12:S1-S12.

2. McWhinney IR. Primary care: core values. Core values in a changing world. BMJ 1998;316:1807-1809.

3. Huygen FJA. Family medicine. The medical life history of families. Nijmegen: Dekker and Van de Vegt; 1978.

4. Van Weel C. Longitudinal research and data collection in primary care. Ann Fam Med 2005;3: S46-S51.

5. Van Weel C. The Continuous Morbidity Registration Nijmegen: background and history of a Dutch general practice database. Eur J Gen Pract 2008;14 Suppl 1: 5-12.

6. FaMe-Net. FaMe-Net reference list. Published March 1, 2021. Available from: https://www.famenet. nl/. Accessed March 1, 2021.

7. Uijen AA, Bor H, Van Boven K. FaMe-Net: twee oude registratienetwerken in een nieuw jasje. Tijdschr Gezondheidswet 2015;93:286-287.

8. Peterson KA, Lipman PD, Lange CJ, Cohen RA, Durako S. Supporting better science in primary care: a description of practice-based research networks (PBRNs) in 2011. J Am Board Fam Med 2012;25:565-571.

9. Lamberts H, Hofmans-Okkes I. Episode of care: a core concept in family practice. J Fam Pract 1996;42: 161-167.

10. Okkes I, Oskam S, Lamberts H, Van Boven K. Episodes of care in Dutch Family Practice. Epidemiological data based on the routine use of the International Classification of Primary Care (ICPC) in the Transition Project. Amsterdam: Academic Medical Center/University of Amsterdam; 2005.

11. De Jongh T, De VH, Grundmeijer H. Diagnostiek van alledaagse klachten. Bouwstenen voor rationeel probleemoplossen. Houten: Bohn Stafleu van Loghum; 2004.

12. Van Boven K, Uijen AA, Van de Wiel N, Oskam SK, Schers HJ, Assendelft WJJ. The diagnostic value of the patient's reason for encounter for diagnosing cancer in primary care. J Am Board Fam Med 2017;30:806-812.

13. Okkes IM, Oskam SK, Lamberts H. The probability of specific diagnoses for patients presenting with common symptoms to Dutch family physicians. J Fam Pract 2002;51:31-36.

14. Houwink EJF, Hortensius OR, Van Boven K, Sollie A, Numans ME. Genetics in primary care: validating a tool to pre-symptomatically assess common disease risk using an Australian questionnaire on family history. Clin Transl Med 2019;8:17.

15. Statistics Netherlands. 2020. Published November 17, 2008. Available from: https://www.cbs.nl/nl-nl/onzediensten/methoden/onderzoeksomschrijvingen/ aanvullende-onderzoeksbeschrijvingen/beknoptemethodebeschrijving-gezonde-levensverwachtingnaar-sociaaleconomische-status. Accessed November 2, 2021.

16. Netherlands institute for health services research. 2020. Available from: https://nivel.nl/en. Published March 1, 2021.

17. Schers H, van Weel C, van Boven K, Akkermans R, Bischoff E, Olde Hartman T. The COVID-19 Pandemic in Nijmegen, the Netherlands: Changes in presented health problems and demand for primary care. Ann Fam Med. 2021;19:44-47.

18. Dijkstra I, Uijen AA, Van Boven K, Schers H. CVRM en belaste familieanamnese. Huisarts Wet 2021;64: $10-15$.

19. Little P, Everitt H, Williamson I, et al. Observational study of effect of patient centredness and positive approach on outcomes of general practice consultations. BMJ 2001;323:908-911.

20. Stolper E, Van de Wiel M, Van Royen P, Van Bokhoven M, Van der Weijden T, Dinant GJ. Gut feelings as a third track in general practitioners' diagnostic reasoning. J Gen Intern Med 2011;26:197-203.

21. Olde Hartman TC, Van Ravesteijn H, Lucassen P, Van Boven K, Van Weel-Baumgarten E, Van Weel 
C. Why the 'reason for encounter' should be incorporated in the analysis of outcome of care. Br J Gen Pract 2011;61:e839-e841.

22. Van Weel C. Primary health care and family medicine at the core of health care: challenges and priorities in how to further strengthen their potential. Front Med (Lausanne) 2014;1:37.

23. Greenhalgh T. Narrative based medicine: narrative based medicine in an evidence based world. BMJ 1999;318:323-325.

24. Fioretti C, Mazzocco K, Riva S, Oliveri S, Masiero M, Pravettoni G. Research studies on patients' illness experience using the Narrative Medicine approach: a systematic review. BMJ Open 2016;6:e011220.

25. Greenhalgh T, Hurwitz B. Narrative based medicine: why study narrative? BMJ 1999;318:48-50.

26. Van de Lisdonk EH, Van den Bosch WJHM, Lagro-Janssen ALM, Schers HJ. Ziekten in de huisartspraktijk. Maarssen: Elsevier gezondheidszorg; 2008.

27. Kyrimi E, Mossadegh S, Tai N, Marsh W. An incremental explanation of inference in Bayesian networks for increasing model trustworthiness and supporting clinical decision making. Artif Intell Med 2020;103:101812.

28. Moons KG, Altman DG, Vergouwe Y, Royston P. Prognosis and prognostic research: application and impact of prognostic models in clinical practice. BMJ 2009;338:b606.

29. Wyatt JC, Altman DG. Commentary. Prognostic models: clinically useful or quickly forgotten? BMJ 1995;311:1539-1541.

30. Hickner J, Green LA. Practice-based Research Networks (PBRNs) in the United States: growing and still going after all these years. J Am Board Fam Med 2015;28:541-545. 


\section{Appendix 1: FaMe-Net: Inception of the World's Oldest PBRN within the Dutch Health care Context}

In the Netherlands, all inhabitants are listed with a family physician (FP), who deals with all bealth problems requiring professional medical care. Dutch FPs provide initial 'entry' to bealth care, and they receive correspondence from other primary care professionals and specialist care. FaMe-Net operates in this context of Dutch bealth care, automatically resulting in a complete medical bistory of all registered patients.

FaMe-Net routinely collects data on the illness presentation on all issues patients present to their FP. It is the oldest, uninterrupted, and still functioning PBRN in the world. FaMe-Net has, from its start in 1967, registered 'complete' morbidity, that is, all morbidity patients present to their FP. It collects data longitudinally. This network is a continuation of the 2 well-known Dutch predecessor PBRNs from which it originated after their fusion in 2013: the Continuous Morbidity Registration Nijmegen (CMR) registering epidemiology since 1967, and the 'Transition Project', registering since 1985.

The CMR has historically had a focus on contextual patient characteristics and their relation to morbidity and was probably unique at that time in routinely collecting data on level of education and family relationships. Huygen, founding father of the CMR and (Dutch) scientific family medicine in general, described morbidity patterns in families in his book 'Family Medicine' published in 1978.*

The fusion resulted in an expanded number of participants, currently approximating 40,000 listed patients in the 7 participating practices. FaMe-Net and its predecessors bave produced numerous publications, including textbooks for medical students and FPs (in training) on clinical reasoning and epidemiology. ${ }^{* \dagger, \ddagger, \$}$ The 2 prior PBRNs applied a different focus in data collection, but both had as crucial core actions to document morbidity in primary care. Since the fusion, all practices code the full spectrum of data recorded within current FaMe-Net, thus combining several characteristics of the predecessor networks. The current article highlights a few unique hallmarks to demonstrate the evolution of the world's oldest PBRN.
The registering practices from the prior CMR are located around the city of Nijmegen in The Netherlands, close to the German border in the eastern part of the country. The network and the registering practices are stable but registering practices may sometimes join or leave the network. In 2019 a new practice opened in the geographically delimited northern area of Nijmegen, across the river 'Waal', where new constructions proceed and joined FaMe-Net. In Spring 2020, an experienced academic general practice in Nijmegen joined as new registering practice for the $P B R N$, with approximately 10,000 listed patients and 6 registering GPs. After fusion to FaMe-Net, the remaining practices from the former Transition Project, close to the Dutch capital of Amsterdam and in the province of Frisland in the north of the Netherlands, fused to 1 larger practice.

FaMe-Net has been able to build a large longitudinal dataset using a uniform and unchanged registration system that will be explained in this article. It contains all FP presented morbidity from 2005 onwards, derived from the former Transition Project data before 2013. Until the end of 2019, this dataset contains 308,000 patient-years and over 2.2 million encounters, with data from 5 family practices (27 FPs) and approximately 30,000 registered patients and continuing registration. FaMe-Net has been shown to provide bigh-quality data derived from an unselected population. The patient population in this network is representative of the general Dutch population in terms of age and sex."

*Huygen FJA (1978) Family medicine. The medical life history of families. Dekker and Van de Vegt, Nijmegen.

${ }^{\dagger}$ De Jongh T, De Vries H, Grundmeijer H (2004) Diagnostiek van alledaagse klachten. Bouwstenen voor rationeel probleemoplossen. Bohn Stafleu van Loghum, Houten.

${ }^{7}$ Van de Lisdonk EH, Van den Bosch WJHM, Lagro-Janssen ALM, Schers HJ (2008) Ziekten in de huisartspraktijk. Elsevier gezondheidszorg, Maarssen.

${ }^{\S}$ FaMe-Net. Published March 1, 2021. Available from: https://www.famenet.nl/.

"Statistics Netherlands (CBS). Published October 30, 2020. Available from: https://www.cbs.nl/en-gb/ figures/detail/37296eng. Accessed November 2020. 


\section{Appendix 2: Context form}

\section{REGISTRATION FORM}

Please use this form to register in our practice.

Surname:

Maiden name (if applicable):

Given name:

Initials:

Date of birth:

Gender: male/female/other,

Street and number:

Postal code:

City:

Telephone:

2nd telephone number:

E-mail:

Citizen Service Number (BSN):

Health insurer:

Insurance number:

New pharmacy:

Previous GP:

Address previous GP:

I hereby consent to the exchange of my patient details with the hospital, pharmacy and the out-of-hours primary care.

I hereby consent to register and to transfer my patient file to the general practice.

Date

Signature

\section{BACKGROUND INFORMATION}

It is important for your GP to have some more background information about you.

We will ask you a number of administrative questions as well as a few questions regarding major life events.

If you prefer not to answer a particular question, please leave this question unanswered and proceed to the next question.

Everything you have written down in this questionnaire will be protected by medical confidentiality and will therefore be treated as confidential.

Filling in this questionnaire will take approximately 5-10 minutes.

\section{What is your country of birth?}

0 the Netherlands

Other, namely:

Both your country of birth and that of your biological parents are of medical importance when it comes to genetic disorders and risk factors.

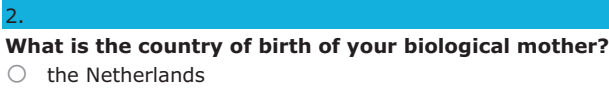

the Netherlands

Other, namely: 
What is the country of birth of your biological father?

the Netherlands

Other, namely:

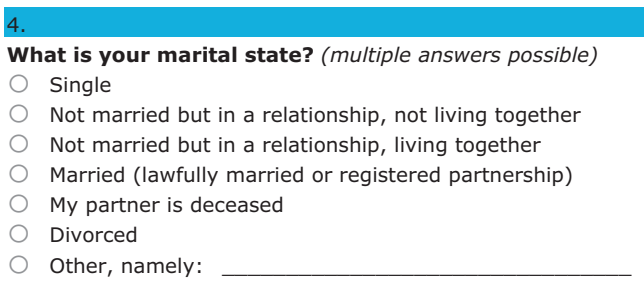

\begin{tabular}{|c|c|c|c|}
\hline Do the following diseases occur in your family? & Yes & No & $\begin{array}{l}\text { Not } \\
\text { known }\end{array}$ \\
\hline $\begin{array}{l}\text { a. Heart diseases in parents, siblings or children before } \\
\text { the age of } 60 \text { ? }\end{array}$ & & & \\
\hline $\begin{array}{l}\text { b. Diabetes type } 1 \text { or type } 2 \text { in parents, siblings, or } \\
\text { children? }\end{array}$ & & & \\
\hline $\begin{array}{l}\text { c. Melanoma (malignant mole) in parents, siblings or } \\
\text { children? }\end{array}$ & & & \\
\hline $\begin{array}{l}\text { d. Colon cancer in parents, siblings or children before the } \\
\text { age of } 50 \text { ? }\end{array}$ & & & \\
\hline $\begin{array}{l}\text { e. Colon cancer in more than one relative in the same } \\
\text { family? }\end{array}$ & & & \\
\hline $\begin{array}{l}\text { f. Prostate cancer in father, brothers or sons before the } \\
\text { age of } 55 \text { ? }\end{array}$ & & & \\
\hline
\end{tabular}

\begin{tabular}{|l|l|l|l|}
\hline Do the following diseases occur in your family? & Yes & No & $\begin{array}{l}\text { Not } \\
\text { known }\end{array}$ \\
\hline $\begin{array}{l}\text { g. Ovarian cancer in mother, sisters or daughters? } \\
\text { h. Breast cancer in parents, siblings or children before the } \\
\text { age of 50? }\end{array}$ & & & \\
\hline $\begin{array}{l}\text { i. Breast cancer in more than one relative in the same } \\
\text { family? }\end{array}$ & & & \\
\hline
\end{tabular}

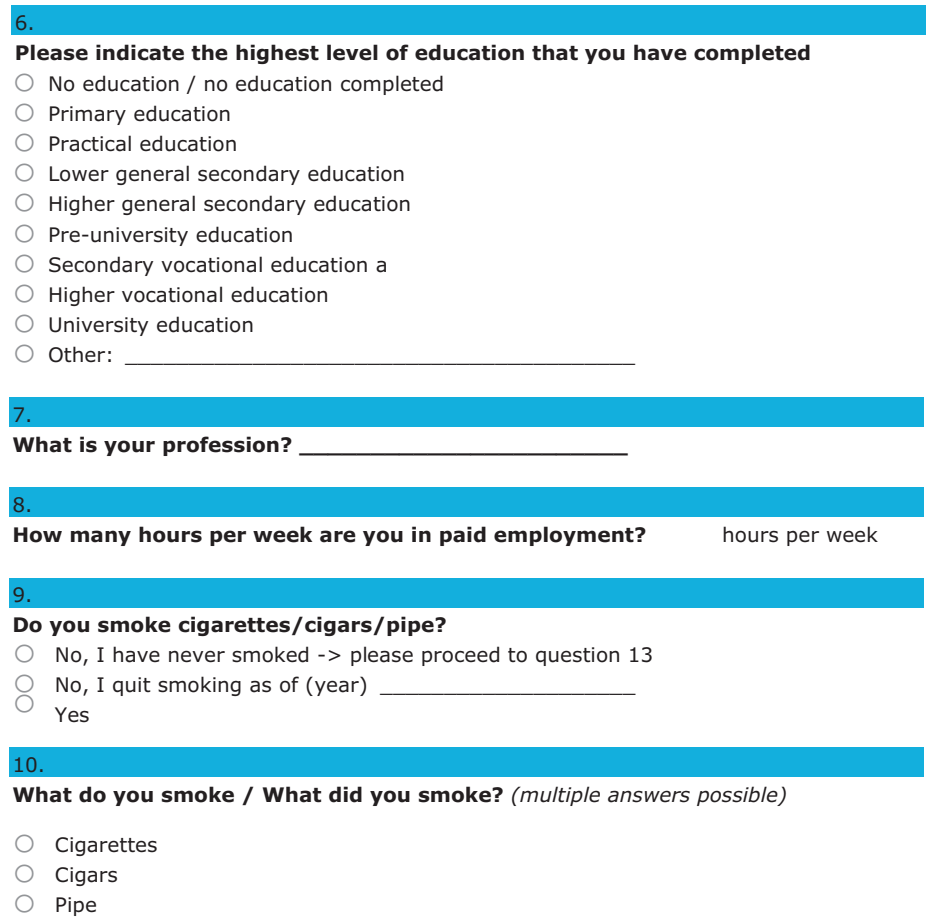

(continued) 


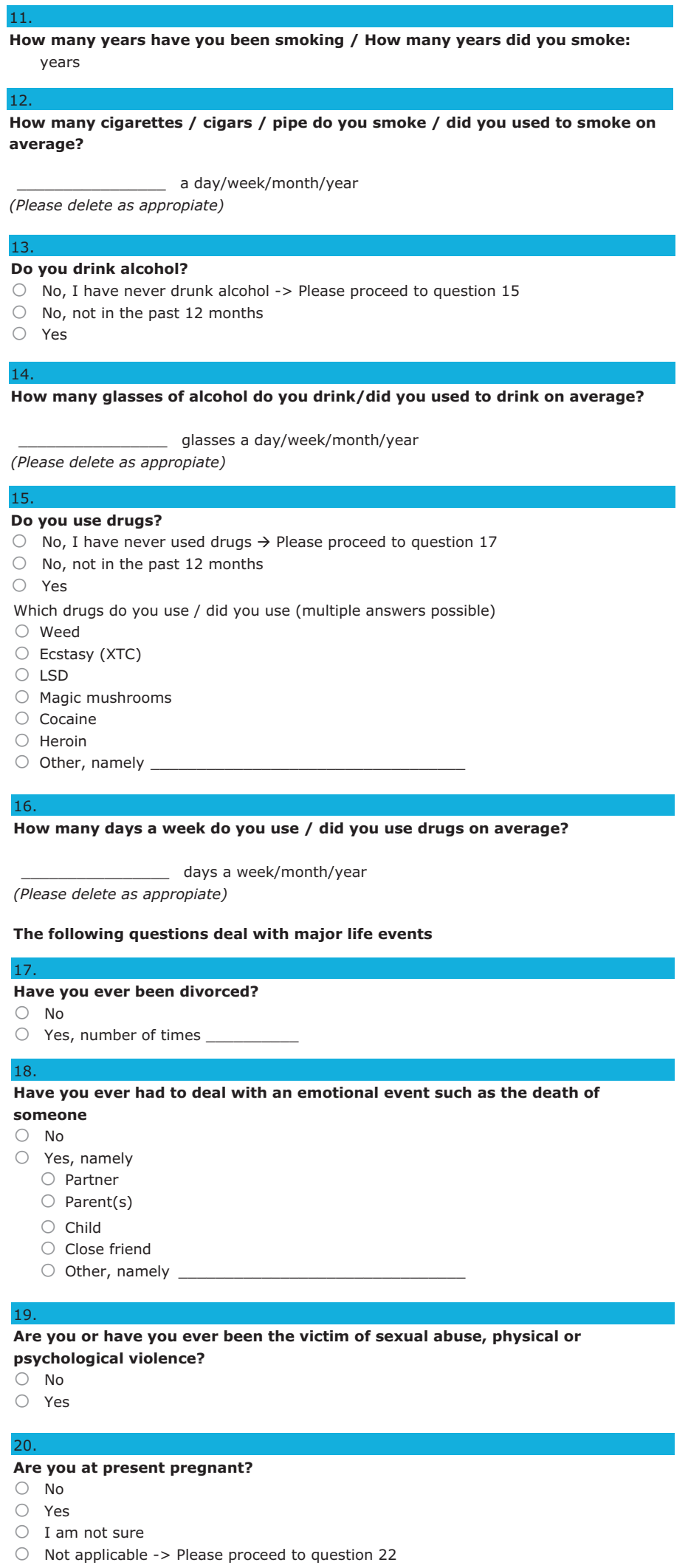

(continued) 
22.

How many biological children do you have?

children

Do you have a responsibility of care for children of whom you are not the biological parent?

Yes, please clarify:

No

Do you have any further (additional) remarks that are of importance to your

GP?

If you have any questions relating to this questionnaire or if you wish to talk about something, please do not hesitate to make an appointment with your GP.

Thank you very much for your time in filling in this questionnaire. 